\title{
Improving Database Vendors' Usage Statistics Reporting through Collaboration between Libraries and Vendors
}

\section{Wonsik Shim and Charles R. McClure}

The article reports the results from the Association of Research Libraries (ARL) E-Metrics study to investigate issues associated with the usage statistics provided by database vendors. The ARL E-Metrics study was a concerted effort by twenty-four ARL libraries to develop and test statistics and measures in order to describe electronic resources and services in ARL libraries. This article describes a series of activities and investigations that included a meeting with major database vendors and the field-testing of usage statistics from eight major vendors to evaluate the degree to which the reports are useful for library decision-making. Overall, the usage statistics from the vendors studied are easy to obtain and process. However, the standardization of key usage statistics and reporting format is critical. Validation of reported statistics also remains a critical issue. This article offers a set of recommendations for libraries and calls for continuous collaboration between libraries and major database vendors.

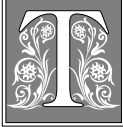

he move to a networked environment has significantly increased the range of services and resources that the library provides its users. The library has become a twenty-four-hour-a-day access point to information services where users obtain services and resources on their terms and when they want such services. Often users do not enter the library physically nor do they interact directly with library staff. The costs of providing these networked services and resources can be significant. As a result, library managers are seeking ways to measure the use of these digital services and resources.

One of the results of the networked information provision environment is that libraries increasingly depend on external providers of academic and scholarly information content and services. Recent statistics estimate that in 20002001, research libraries spent, on average, 16.25 percent of their materials budget on electronic resources, a sharp increase from a mere 3.6 percent in $1992-1993 .^{1}$ This information has traditionally existed in the library as subscription print journals,

Wonsik Shim is an Assistant Professor in the School of Information Studies at Florida State University; email: wshim@lis.fsu.edu. Charles R. McClure is the Francis Eppes Professor and Director of the Information Use Management and Policy Institute in the School of Information Studies at Florida State University; e-mail: cmcclure@lis.fsu.edu. 
print indexes and abstracts, books, and so on. However, there is a big difference in terms of ownership and control between traditional information contents and digital information contents.

\section{... the provision of usage statistics by electronic content providers is problematic at best.}

With physical media, the library owned the objects and controlled their use. For example, the library catalog-be it card catalog or online catalog-represented what the library owned and could make available to its users. But with electronic media, the library is only one of many access points to the information resources. As a result, the library has much less control over use. ${ }^{2}$ The library catalog now includes many pointers to external information sources that, in some cases, may no longer exist when the user tries to access them.

Figure 1 depicts a simplified view of the differences between the traditional library environment and the networked library environment characterized by the Internet as the primary information delivery medium and the growing presence of external electronic information resources and services in the library.
In the traditional library, most library materials were housed in a physical library building, and users typically needed to come to the library to use its materials and services. ${ }^{4}$ Availability was an important concern because of the physical characteristics of the materials. In the networked library, however, library materials and services increasingly reside outside the physical library building. Libraries now depend, in large measure, on the publishers of electronic journals (e.g., Elsevier's Science Direct and Academic Press's IDEAL), electronic content aggregators (e.g., Ebsco and Gale), and other electronic information providers to meet user demands for resources and services. ${ }^{5}$ Availability has become less an issue in the networked library environment because the electronic medium allows several people to use the same material at the same time. ${ }^{6}$

On an experiential basis, many academic librarians describe the use of their networked information services with terms such as "exponential growth" or "we can't keep up with demand." At the same time, a number of academic libraries have seen stagnant or declining statistics of traditional indicators of library service, such as turnstile counts, in-house reference transactions, and circulation.

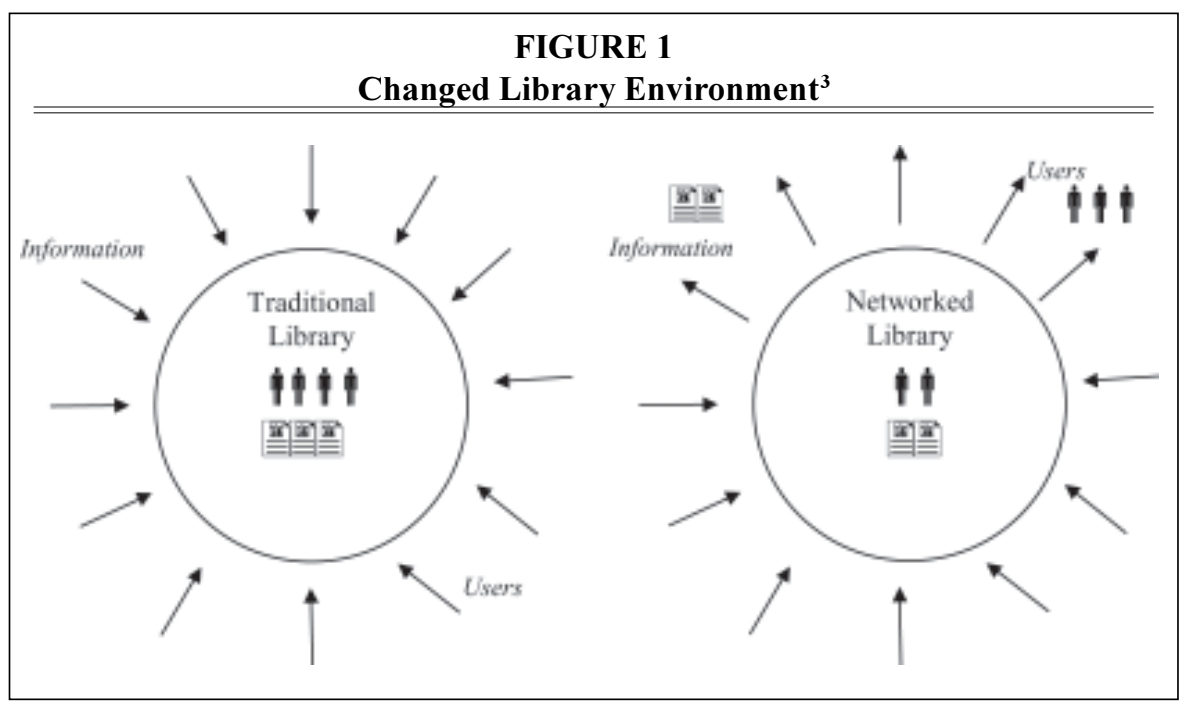


Librarians need reliable and accurate statistics that will allow them to make good resource allocation decisions (e.g., costbenefit analysis, contract negotiation, justification of expenditure), meet user needs (e.g., identifying barriers of access, understanding user behaviors), and develop strategic plans (e.g., user education, peer comparison) for the development and operation of electronic services and resources.

Although some progress has been made over the past several years, most notably, the guidelines produced by the International Coalition of Library Consortia (ICOLC), the provision of usage statistics by electronic content providers is problematic at best. $^{7}$

This article focuses on the problem of acquiring and using the statistics provided by external, fee-based electronic content providers and describes the work done in the Association of Research Libraries (ARL) E-Metrics Project to standardize the usage statistics and promote dialogue among database vendors and research libraries. ${ }^{8}$

\section{Previous Work}

The growing presence of electronic information resources and networked services prompted interest and research in developing statistics and measures to describe the emerging information environment. The most relevant work is a manual published by the ALA in 2001. ${ }^{9}$ Written by John Carlo Bertot, Charles R. McClure, and Joe Ryan, the work is based on Developing Public Library Statistics and Performance Measures for the Networked Environment, a research project funded by the Institute of Museum and Library Services (IMLS). Intended primarily for public library managers, the manual not only contains step-by-step procedures to collect some key usage statistics but also provides a set of issues that library administrators need to consider in collecting and using those statistics. Many of the proposed statistics can be easily transferred into an academic library setting.
In an article published in 2000, Carol Tenopir and Eleanor Read offered an example of cross-institutional analysis of database use. ${ }^{10}$ Using data from a vendor for fifty-seven academic institutions, Tenopir and Read found that regardless of the type of academic library, user demands are concentrated on a fairly predictable span of time- - "early in the week, at midday, in the month when term papers are due." The authors also concluded that, compared with other electronic media such as chat rooms and general Internet resources, students underutilize electronic library databases. At the individual institution level, Deborah D. Blecic, Joan B. Fiscella, and Stephen E. Wiberley Jr. identified ways that libraries can use vendor-supplied usage statistics to understand the scope of use. ${ }^{11}$ Sally A. Rogers also has provided a good comparison of print and e-journal usage at Ohio State University. ${ }^{12}$ Recognizing the need for ways to compare patron usage of electronic and print materials, Kathleen Bauer proposed the use of indexes to combine multiple usage indicators of both electronic and print resources. ${ }^{13}$

A recent compilation by $\mathrm{McCl}$ ure and Bertot has provided an overview of a wide array of issues surrounding the evaluation of networked information services in several different contexts, including usage statistics from database vendors. ${ }^{14}$ As mentioned earlier, the ICOLC guidelines are widely recognized as the de facto standard regarding usage statistics supplied by database vendors.

Finally, the discussion of usage statistics of database vendors is not complete without mentioning two very active mailing lists that deal with the topic: Library License (liblicense-1@lists.yale.edu), hosted by the Yale University Library; and the Electronic Resources in Libraries list (eril-1@listserv.binghamton.edu), hosted by the Binghamton University Library. Although these mailing lists do not cover vendor statistics exclusively, there have been a considerable number of postings and threads on both regarding the topic. These mailing lists also have been used 
as a catalyst to formulate the library community's response to major challenges from database vendors.

The current work focuses on issues related to acquiring, processing, and using vendor usage statistics at research libraries under the ARL E-Metrics Project. It is important to point out that the E-Metrics Project is one of many initiatives that are working toward establishing standardized, comparable statistics for electronic contents and services. However, the ARL E-Metrics Project is unique in that it is a cooperative effort among a large number of research libraries and that it seeks the participation of major database vendors in attempting to find solutions.

\section{ARL E-Metrics Project}

Usage statistics in the context of electronic subscription-based databases mainly refer to the indicators of the volume of user access to the electronic resources and services available from database vendors. Examples of those indicators are a count of sessions in a specific database, the time per session in a specific database, the count of searches in a specific database, and the count of full-text downloads per time period per database. In addition, usage statistics can show a variety of information, including success or failure of user access (e.g., turn-aways per time period per specific database), user access methods (e.g., telnet versus browsers), access levels of one institution compared against peer institutions, cost of access (e.g., cost per downloaded item), and other items pertaining to user behaviors.

According to a survey conducted with the participants of the February 2000 ARL Project Planning Session on Usage Measures for Electronic Information Resources, held in Scottsdale, Arizona, the following problems are associated with usage reports from database vendors: ${ }^{15}$

- Reports do not provide detailed information about usage. For example, many vendors did not provide usage figures by journal or database title.

- Reports are inconsistent. For example, vendors use their own terminolo- gies and do not provide adequate explanations to understand the reported statistics.

- Reports are not comparable. Because usage reports come in different formats and contain different statistics, it is impossible to compile accurate statistics within the library and to compare with other libraries.

However, the biggest problem with usage reports is that many vendors simply do not provide any data at all.

The ARL E-Metrics Project was a concerted effort by selected members of the research library community to investigate various issues and problems related to collecting and using data on electronic materials and services. The project, which began in April 2000 and finished in December 2001, was funded by a group of twenty-four ARL libraries. Figure 2 identifies the project's participants.

One of the aims of the E-Metrics Project was to engage in a collaborative effort with selected database vendors to establish an ongoing means of producing selected descriptive statistics on database use, users, and services. A complete project description, project reports, and the data collection manual are available at the ARL E-Metrics Project site at http://www.arl.org/stats/ newmeas/emetrics/index.html.

The E-Metrics Project should be viewed in the context of a number of related initiatives, both national and international, that are under way to assist libraries in assessing their networked resources and services. Although these initiatives take different approaches, focus on different types of libraries, and work within various operating environments, they all focus on developing library electronic statistics and performance measures. These efforts include:

- International Coalition of Library Consortia (ICOLC): Since the mid1990s, this international coalition of libraries-predominantly academichas been working toward a standard set of definitions for subscription online contents. It published the first guidelines in November 1998 (see 


\section{FIGURE 2}

ARL E-Metrics Project Participants

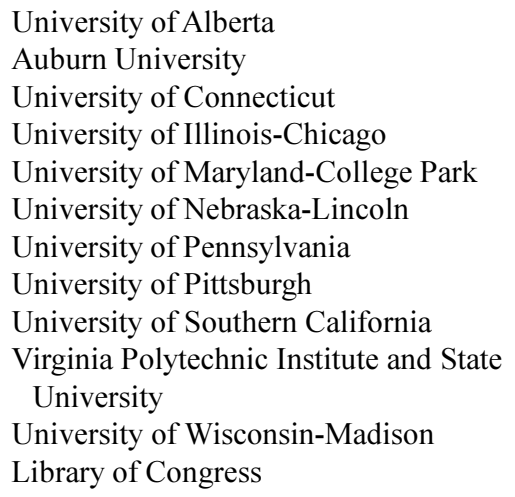

Arizona State University

University of Chicago

Cornell University

University of Manitoba

University of Massachusetts

University of Notre Dame

Pennsylvania State University

Purdue University

Texas A\&M University

University of Western Ontario

Yale University

New York Public Library, The Research Libraries http: / / www.library.yale.edu/consortia/webstats.html) and a revised version in December 2001 (see http:// www.library.yale.edu / consortia / 2001webstats.htm).

- National Information Standards Organization (NISO): NISO is updating its Z39.7-Library Statistics Standard to include network services and resources statistics and performance measures. The draft standard was completed in 2002 (see http:/ /www.niso.org/emetrics/current/ complete.html).

- National Commission on Libraries and Information Science (NCLIS): Over the years, NCLIS has continued its work in standardizing online database usage statistics and reporting mechanisms. This project largely focuses on the public library environment (see http://www.nclis.gov).

- Institute of Museum and Library Services (IMLS): IMLS sponsored a project to develop national network statistics and performance measures for public libraries. The project resulted in a network statistics manual for public libraries. ${ }^{16}$

- Project COUNTER (Counting Online Usage of Networked Electronic Resources): COUNTER is supported by a group of publishers, library associations, and other library-related national bodies whose primary aim is to formulate an international code of practice (COD) governing the recording and reporting of usage statistics.
The release of the first COD is expected by the end of 2002 (see http:// projectcounter.org).

- National Clearinghouse for Library and Information Center Networked Statistics: Proposed by Charles R. McClure and his associates at the Information Use Management and Policy Institute, Florida State University, establishment of the clearinghouse will facilitate the sharing and dissemination of primary data, tools, education, and research regarding statistics of networked resources and services (see http://www.ii.fsu.edu).

It also appears that different vendors use different counting mechanisms.

One important issue regarding these initiatives is the extent to which the initiatives and organizations coordinate with one another. For a host of reasons, including vendor cooperation, library reporting requirements, and library management needs, more coordination and cooperation is necessary throughout these projects. The authors are involved in a number of projects mentioned above and, to the extent possible, will cooperate with other groups.

\section{ARL Meeting with Database Vendors}

A meeting with a select group of large database vendors occurred on March 14, 2001, in conjunction with the ACRL an- 
nual meeting in Denver. The goal of this meeting was to engage the community of vendors, publishers, and libraries in building consensus for reporting data on the use of vendor databases and to promote an understanding of what can and cannot be done vis-à-vis the provision of data from the vendor community. The meeting served as a discussion forum for:

- sharing information about the development and standardization of selected statistics that describe users and uses of databases;

- reaching agreement on the important data elements and definitions;

- engaging vendors in a test of data elements being designed;

- understanding the issues that affect vendor-supplied statistics describing database use and users;

- developing a process so that the library community and the vendor community can work together in developing and standardizing a core set of statistics.

A total of nine vendors attended the meeting, as shown in figure 3 .

During the meeting, both the vendor and the library representatives agreed that the reported statistics should be based on the ICOLC guidelines. It was noted that the market is increasingly diversified in terms of business models, content provided by vendors, and other factors. Accordingly, developing a standardized set of statistics that cover all of these will continue to be a challenge.

Everyone agreed that technologies and technology changes have a lot to do with what and how statistics can be collected and reported. For instance, Z39.50 clients do not allow statistics to be collected. Solutions, such as digital certificates, also are technology based. However, in most cases, the costs of buying and implementing these technologies may outweigh any attempt to justify their use to produce more reliable and detailed data.

It also appears that different vendors use different counting mechanisms. As a result, the compiled statistics have limited reliability and validity. Additional investigation into these and related questions is needed.

\begin{tabular}{|l|}
\multicolumn{1}{|c|}{$\begin{array}{c}\text { FIGURE } 3 \\
\text { Database Vendors } \\
\text { Attending the ARL Meeting }\end{array}$} \\
\hline \hline Elsevier/ScienceDirect \\
netLibrary \\
OCLC/FirstSearch \\
JSTOR \\
Pro Quest \\
Ovid \\
Lexis-Nexis \\
Gale Group \\
EBSCO
\end{tabular}

Overall, the meeting was very useful in that it brought libraries and vendors together and established a dialogue. ${ }^{17}$ The meeting also was a necessary first step for the upcoming field-testing of proposed statistics developed by the E-Metrics study team. As a result of the meeting, all of the vendors present agreed to participate in the vendor statistics field-testing.

\section{Vendor Statistics Field-testing}

The primary goal of the field-testing was to assess usage statistics from major database vendors in terms of comparability of statistics and their definitions, breakdown of data, and report formats.

\section{Methodologies}

Invitations were sent to several vendors, including those that participated in the ARL meeting. All the vendors contacted, twelve in all, agreed to participate in the field-testing. The invitation explained the goals and objectives of the field-testing and provided a brief summary of expected deliverables from each participating vendor.

A set of field-testing guidelines was developed and an electronic copy distributed to the vendors. In addition, project participants (libraries) were contacted and their participation in the field-testing was solicited. Because not all fieldtesting libraries subscribed to all of the services, three or four vendors were assigned to each library based on its subscription matrix. The intent was to allevi- 
ate the burden on the libraries of evaluating too many vendor reports. In addition, from the standpoint of vendors, it seemed to make sense to concentrate on a few libraries rather than all of the libraries subscribing to their services.

The guidelines asked specifically for four deliverables from each vendor:

1. a monthly report (April 2001) in a standardized text format (specific guidelines were given for data elements and their arrangement);

2. a detailed, step-by-step description of the process used to collect the statistics, including the rules and assumptions applied in the process; file;

3. a monthly (April 2001) raw data log

4. issues and suggestions related to providing usage statistics.

The vendors were asked to send the field-testing data to their assigned libraries and to the authors at Florida State University by the last week of May 2001. A separate evaluation questionnaire was developed and distributed to the fieldtesting libraries.

\section{Field-test Findings}

Vendor statistics change constantly and can therefore be considered a moving target. The information presented here is for illustration purposes only and may not correctly reflect the current practices and offerings of the database vendors mentioned in this report.

A total of eight vendors participated in the fieldtesting. Table 1 shows the data formats in which the field test reports were provided by the vendors and the availability of documentation received from the vendors with regard to the definitions of the statistics provided and information on how data were collected, filtered, and aggregated.

The majority of vendors investigated provided usage reports in a text format as well as other formats. Compared with the results from the vendor statistics analysis during the E-Metrics Project, the evidence indicates that vendors have made good efforts, especially in the area of making documentation available. ${ }^{18}$ Many vendors simply did not have any documentation about usage statistics at all when the authors initially analyzed their reports. However, many of the vendors' documentation did not provide enough details concerning the definitions of reported statistics to aid in an understanding of those statistics.

Table 2 shows key ICOLC statistics included in each vendor's field-testing report. It is important to understand that no attempt has been made to validate compliance with the ICOLC guidelines. Aside from the ICOLC guidelines, there are many instances where the same statistics from different vendors are not equal measures. An obvious example is how vendors apply time-out parameters to compute session counts. Vendor documentation indicated a wide range of timeouts (e.g., Gale, 6 minutes; Ebsco, 10 minutes; and Science Direct, 30 minutes).

A more serious case results from the fact that similar vendors use different methodologies to count the same user activity. As a result, even the most seemingly simple statistics, such as searches and items requested, might not be duly compared. Is a search to multiple database packages (as in the case of Gale or

\begin{tabular}{|c|c|c|}
\hline \multicolumn{3}{|c|}{$\begin{array}{c}\text { TABLE } 1 \\
\text { Vendor Statistics Field-testing Participation }\end{array}$} \\
\hline Vendors & Data Format & $\begin{array}{l}\text { Availability of } \\
\text { Documentation }\end{array}$ \\
\hline Academic Press & txt, Excel & n.a. \\
\hline Pro Quest & Excel, txt, PDF & Yes \\
\hline Ebsco & txt & Yes \\
\hline Gale Group & $\operatorname{csv}$ & Yes \\
\hline Lexis-Nexis & zip (csv), Word, txt & Yes \\
\hline NetLibrary & zip (txt), csv & Yes \\
\hline Science Direct & txt & Yes \\
\hline SilverPlatter & $\operatorname{csv}$ & n.a. \\
\hline
\end{tabular}


TABLE 2

Key ICOLC Statistics Included in the Vendor Reports (by vendor)

\begin{tabular}{llccc}
\hline \hline Vendors & Items Requested & Searches & Sessions & Turn-aways \\
\hline $\begin{array}{l}\text { Academic Press/ } \\
\text { IDEAL }\end{array}$ & $\begin{array}{l}\text { Full text, reference, abstract, } \\
\text { table of contents }\end{array}$ & Yes & Yes & n/a \\
Pro Quest & Full text, abstract, citation & Yes & No & n/a \\
Ebsco & Full text, abstract & Yes & Yes & n/a \\
Gale Group & $\begin{array}{l}\text { Full text, citation, abstract, hits, } \\
\text { views, print station }\end{array}$ & Yes & Yes & Yes \\
Lexis-Nexis & Full text, document retrievals & Yes & No & n/a \\
NetLibrary & $\begin{array}{l}\text { Page view, browse, checkout, } \\
\text { dictionary use }\end{array}$ & Yes & Yes & Yes \\
Science Direct & Full text, abstract & & & Yes \\
SilverPlatter & Full text, abstract & Yes & Yes \\
n/a: Not applicable & & Yes & Yes & Yes \\
\hline
\end{tabular}

Ebsco) counted as a single search or as a separate search for each database chosen? Is browsing a secondary database such as author, subject, or journal list counted as a search or a menu selection? Does the vendor take into consideration multiple requests for the same document in a short time period (say, less than 10 seconds) and treat them as one request or multiple requests? Is clicking the next button to retrieve the next set of results counted as separate search? The list of questions goes on and on. The answers to all of these questions can significantly inflate or deflate the reported usage counts. Furthermore, what happens if a vendor changes its counting methodology and does not disclose it?

There is widespread suspicion among librarians that even the identically labeled statistics are counted differently. A close examination of vendor documentation provided seems to suggest that the suspicion is not unfounded. Indeed, the answers to the above-mentioned questions differ among vendors. Another important problem has to do with the fact that most vendors do not provide detailed information to libraries, making it difficult for librarians to determine whether two comparable statistics from two different vendors refer to the same thing and can be compared accordingly. All of these issues seriously undermine the usefulness of usage statistics and threaten the validity of data. (The issue of validity is addressed later in this article.)

As a result of the fact that the types of content available through vendors are increasingly diverse and the terms referring to information items have not been fully standardized, a cross-comparison of the items-requested statistic can be difficult. For example, netLibrary, which is gaining increased presence in research libraries, does not lend itself easily to the kinds of statistics with which we are now familiar. This presents a challenge if libraries try to aggregate the total number of items accessed for cross-vendor comparison or to gauge the total amount of information transfer from licensed materials available at their institutions. 
The turn-away statistic has been useful in determining whether to increment the number of simultaneous user licenses. However, the statistic applies only to those vendors that have such a restriction. Table 2 shows that out of the eight vendors, only three have simultaneous user limits and all three report the turn-away measure.

Table 3 shows a breakdown of reported statistics according to the ICOLC-recommended categories. It also lists other breakdown categories that the vendors reported. It appears that vendors, in general, satisfied the title-level (journal, database, or book) breakdown requirement. The IP (Internet protocol) breakdown requirement also was being generally respected. But in all cases, the statistics were lumped at the subnet (a group of IP address block) level rather than at the individual IP address level. The tabulation might not have been included in summary statistics anyway because it can be made available in log files. Unfortunately, most vendors were unable to furnish log data files because of technical and legal concerns. Half the vendors currently provide some time-related breakdowns.

\section{Libraries' Evaluation of Vendor Reports}

Overall, libraries reported that the data files were easy to read and process. The majority of libraries used Microsoft Excel to import and display data files. In one case, a vendor sent part of the data files in pdf format, which forced the recipient libraries to enter the numbers manually. The results show that libraries would prefer data formats, notably, text formats that can be easily imported into data analysis programs such as Excel and Lotus 1-2-3 without having to spend extra time and effort to manipulate or enter the data.

Although all participating libraries at least opened the data files, only a few attempted to analyze the data. There seemed to be several reasons why libraries were hesitant about in-depth analysis of data. One library commented that it did not test the data because they were the

\begin{tabular}{|c|c|c|c|c|}
\hline \multicolumn{5}{|c|}{$\begin{array}{l}\text { TABLE } 3 \\
\text { Breakdown of Statistics in the Vendor Reports (by vendor) }\end{array}$} \\
\hline Vendors & $\begin{array}{l}\text { By Journal or } \\
\text { Database Title }\end{array}$ & IP & Time/Day & Other \\
\hline $\begin{array}{l}\text { Academic Press/ } \\
\text { IDEAL }\end{array}$ & Journal title & Yes & No & \\
\hline Pro Quest & Database title, journal title & Yes & Time & Client ID \\
\hline Ebsco & Database title & Yes & No & $\begin{array}{l}\text { Group and } \\
\text { profile ID }\end{array}$ \\
\hline Gale Group & Database title, journal title & No & Time, Day & \\
\hline Lexis-Nexis & Database title & Yes & Time, Day & \\
\hline NetLibrary & Book title & Yes & Time, Day & \\
\hline Science Direct & Journal title & Yes & No & $\begin{array}{l}\text { Subscribed } \\
\text { versus non- } \\
\text { subscribed }\end{array}$ \\
\hline SilverPlatter & Database title & No & No & $\begin{array}{l}\text { Peak time and } \\
\text { duration }\end{array}$ \\
\hline
\end{tabular}


summary data and not the raw data the library expected from the field-testing. The following comment from another library also explains why libraries have not done further analysis: "We currently place raw vendor statistics on our staff intranet and do not compile them for comparison purposes, as we have yet to define what statistics and what format would best suit our institutional needs for such a compilation."

\section{The market for electronic content providers is becoming more diverse and complicated, and the types of statistics that best serve libraries in this changing environment need to be considered.}

At least one library reported specifically how it processed the field-testing data. For each vendor analyzed, the library compared the session counts from the library redirect page (all requests to external vendor databases pass through a Web page that counts how many times different databases are accessed) and the vendor report. This produced, for each database, a rough idea of what portion of attempted log-ins (sessions) originated from people who bypass the library database Web page. The library also calculated the estimated cost per article viewed and the distribution of articles viewed by title, which confirmed that 25 percent of the titles account for 80 percent of articles viewed for the particular database.

The field-testing instructions provided guidelines in terms of essential data elements, data arrangement, and file format. Contrary to the authors' expectations, all of the vendors simply repackaged their monthly usage reports and submitted them to the libraries. Therefore, the only practical difference between the field-testing report and the report that libraries accessed from the vendor Web site in a normal situation was that libraries received the data files directly from the vendors instead of retrieving them from the vendor Web sites. Several libraries appreciated the fact that they could receive data files in text format, which is much easier to handle than, say, HTML format. Another minor difference was the availability of data definitions and statistics collection processes from some of the participating vendors. In some cases, this was the first time that explanations were available to the libraries. Typically, documents that contain definitions of statistics and other background information, if they are available, are provided on the vendors' Web sites.

Even when the sets of data were available from vendors, it was difficult for the libraries to do valid comparisons of the data because of insufficient descriptions of data definitions and limited explanation of how the data sets were collected and summarized. Many libraries feared that, without explanatory information on what each data element in the vendor reports meant and how the counts were filtered, such a comparison would have been faulty at best. This suggests that until there is a satisfactory degree of assurance that the statistics provided by the different vendors-based on the documentation they provide-are consistent enough for cross-comparison, libraries will not commit major resources in an attempt to compile vendor data into a standardized format or repository.

Another problem with comparing data from multiple vendors was the inconsistent data formats. The task of combining data fields and adjusting data arrangement from even three or four vendors proved to be extremely time-consuming. What libraries want is a standardized usage report containing common data elements and arranged in a predetermined, agreedupon order that is provided separately from vendor-specific data elements or additional data. Even the different placement of field headings, in a column or in a row, requires special handling by the libraries.

The majority of respondents said that the data provided by these vendors are "necessary and valuable." They liked the fact that the data are "very straightforward and easy to use" and, more important, that they provide some indication of 
the extent to which subscription-based services are being utilized. Of course, the relative value depends on the quality of data and the importance of the database to the library (e.g., the amount of money the library spends for a particular database as compared with other databases to which they subscribe).

Although the majority of libraries believed that the usage reports provided by the individual vendors are useful, some questioned the cumulative value of usage reports combined across vendors. Given the fact that typical ARL libraries deal with several dozen database vendors, normalizing the data, in the current forms, from these vendors will require a prohibitive amount of effort.

Usage reports deal almost exclusively with the specific use of vendor databases in terms of frequencies (e.g., searches and sessions), duration (e.g., connection time), and amount of information transfer (e.g., items requested) while largely ignoring another dimension that many libraries consider very important-information about user behavior. The current usage metrics provide information about user behavior to a degree, but not at the level many libraries would hope.

To be useful, information about user behavior will need to be correlated with individual user profiles. But the current environment for database access, which is heavily rooted on IP-based authentication, does not permit the kind of data collection that libraries expect. Although there is a desire to receive more detailed information about user behaviors, it conflicts with the current practices and the libraries' concern about user privacy.

Optimally, vendors would provide an option that allows libraries to access raw data $\log$ files that have sufficient information for useful analysis and standardized definitions, and that are collected consistently over time. Unfortunately, many vendors were unable to provide log data files because of technical, legal, or other concerns.

Because the field-testing dealt with only one month's data (April 2001), it is difficult to know if what was collected is typical. However, the authors have not heard from the field-testing libraries of any unusual discrepancy between the field-testing data and data they received before the field-testing. The authors realize that just comparing data from the same vendors will not provide a satisfactory answer to collecting accurate, reliable, and standardized data.

During the course of writing this report, the authors came across an e-mail message from a major database vendor acknowledging errors in its usage reports. This suggests that libraries are not in a good position to know what exactly goes into the vendor reports. Some unusual numbers or patterns are relatively easy to identify, but consistent under- or overcounts are harder to detect.

The authors believe that the data provided from the vendors studied are easy to obtain and manipulate. Most vendors offer several data formats, including text format (e.g., comma-separated file) and spreadsheet format (e.g., MS Excel), in addition to standard HTML format for easy viewing in Web browsers. Also, many vendors offer an ad hoc report generation facility whereby libraries can customize the fields and set desired time periods they want to examine. However, processing vendor reports from multiple vendors may become a burden on libraries in terms of time and staff efforts because the formats and data arrangements vary considerably from vendor to vendor.

Dealing with vendor usage reports raises a number of other issues. The market for electronic content providers is becoming more diverse and complicated, and the types of statistics that best serve libraries in this changing environment need to be considered. Companies such as netLibrary did not even exist when the ICOLC guidelines were first drafted. A related issue is the effect of mega-mergers taking place in the electronic content providers' market and how these mergers will affect statistical reporting.

For the most part, libraries have relied on the ICOLC guidelines as the de facto 
standard for usage statistics for licensed materials. Indeed, the guidelines brought the issue of usage statistics to full view for many practicing librarians and database vendors. Although most vendors included in the study claimed a high level of compliance with the guidelines, some librarians remain skeptical, citing the differences in the way statistics are collected by different vendors (e.g., different time-outs) and the lack of concrete documentation. The ICOLC guidelines are concerned mainly with defining basic usage statistics and do not contain detailed information that can be used to validate whether the vendor reports adhere to the standard. In addition, the library community may have different opinions about how statistics should be counted. What level of specificity are we pursuing in the standardized reports? And who is going to ensure that a vendor report meets the accepted standard?

The validity of usage statistics is a critical issue that needs to be addressed seriously. First, there should be more detailed information to analyze the validity of reported statistics from database vendors. The current documentation, albeit improved, is simply not adequate. In this regard, Project Counter is an important initiative because it attempts to define an agreed-upon code of practices. All related parties need to work together to draw up the specifics of the practices to dispel the persistent suspicion that even the same statistics are counted differently. For this to happen, libraries, publishers, and aggregators need to continue a healthy dialogue regarding their expectations. Vendors need to be more forthcoming in the discussion and better describe what they do and how they do it in usage reporting. Because practitioners themselves sometimes do not agree on what is valid, the library community needs to determine what a valid metric is. Establishment of the National Clearinghouse for Library and Information Center Networked Statistics described earlier can help formulate consensus among practitioners. Finally, an external validation service or organization can be considered as a part of the solution. The validating service then would enforce compliance to industry standards and monitor actual use. The authors mention this simply as a possibility in the long term and suggest that it be thoroughly examined before being put forward for implementation.

This study has not dealt with issues related to usage reporting in consortial arrangements. As those are becoming very common in research libraries, librarians will need to make sure that the individual members involved in the consortia receive the same level of usage statistics for their institutions as in individual site-licensing agreements.

Usage statistics currently provided by vendors give useful information regarding the utilization of external subscription-based information services. Libraries use the data for a variety of purposes: usage trends over time, justification for expenditures, cost analysis, modification of service provision, and so on. Related to the issue of the value of the data is the trustworthiness (reliability) of the data. And, as discussed earlier, there also is some concern about the lack of user-related information in usage statistics.

\section{Recommendations}

Based on the findings of this study, the authors make several suggestions that may be useful for ARL libraries (and perhaps other libraries) to consider in dealing specifically with vendor statistics, including:

- Focusing data analysis on high-impact databases: Libraries should not treat all databases equally when it comes to data analysis. Because of inconsistencies in data elements and report delivery, it is difficult to normalize usage statistics from all vendors who report data. Instead, libraries need to investigate the usage patterns of "major" databases, whatever those might be locally, and ways that improvements can be made in terms of access and use of materials.

- Collecting locally obtainable data for external databases: Although libraries need to depend on database vendors for usage 
statistics, they have several ways (e.g., through redirect page counters for licensed databases or through proxy server logs) to capture at least partial information on user access to the external databases (e.g., attempted log-ins). This kind of internal data helps libraries spot-check the reliability of vendor-supplied usage statistics. Moreover, because the data will be under the control of libraries, they will be more consistent than measures reported by different vendors.

- Keeping track of aggregate key statistics and use them: Libraries often find themselves in need of gross figures of user access to external licensed databases for various internal and external reporting. The aggregate numbers are good indicators of overall trends in user demand for, and access to, external databases. It is important to keep some level of consistency in the way the gross figures are calculated and reported. One way to maintain consistency is to gather data from the same pool of database vendors or database titles over a specified period of time (e.g., Total number of searches conducted in existing licensed databases grew by $20 \%$ in 2000 to $1,200,000$ as compared to the 1999 total of $1,000,000$ searches. The data are based on the same thirty-five vendors that report the statistic.).

- Validating reliability: The library community needs to consider concrete ways (e.g., third-party validation) to ensure consistent and reliable reporting from vendors.

- Demanding documentation: Libraries should demand better documentation of the data collection and filtering process from the various vendors. Such documentation should describe how the sets of data are collected and defined, and discuss any issues or concerns in the reporting of these data to libraries.

- Organizing the library for data collection, analysis, and working with the vendors: Many libraries simply lack adequate staff, or the staff members lack adequate knowledge and training, to work effectively with the statistics and information that some of the vendors can supply. Li- brary staff need to have an understanding of the statistics and to know how to manipulate the files and how to organize and report such data. In addition, the library needs to be able to commit organizational resources to working with and using such vendor statistics.

The use of different system parameters (e.g., time-out), the application of different assumptions about user behavior (e.g., how to treat or count multiple clicks on the same document within a session), and the lack

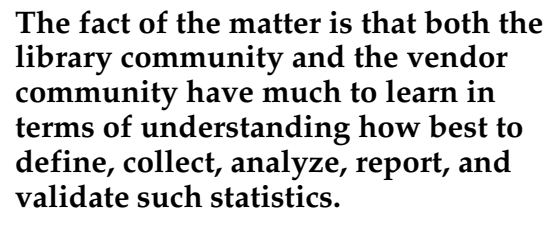

of adequate explanation in vendor documentation regarding specific definitions and data collection and filtering processes all contribute to the reporting problem. The comprehensive standardization of usage statistics and data delivery methods (e.g., file format and data arrangement) cannot be easily achieved in the short term. These are long-term goals toward which vendors and libraries need to work together. The ARL community should continue to make progress in this area by working among themselves and with the database vendor community. Therefore, the authors recommend that comparisons be limited to data from the same vendors or data that are known to be collected, defined, and reported similarly.

The authors strongly recommend that vendors report standardized usage statistics, such as those recommended by the ICOLC and those defined in the final manual that resulted from the project. ${ }^{19}$ These should appear in the standardized column and row arrangements and include a separate report that contains any additional vendor-specific data.

\section{Continuing the Momentum}

ARL libraries have needed consistent, comparable, easy-to-use, and useful usage statistics from content providers (da- 
tabase vendors) ever since they embraced the notion of maintaining statistics on the use of external licensed materials. The ARL E-Metrics Project provided an opportunity for the ARL community to look at the issues and problems related to vendor usage reporting in a more systematic way and to begin working toward developing more useful reports. However, much more work remains in this area.

Members of the study team found that some library staff had little knowledge about the vendor statistics, had limited training in being able to manipulate and analyze the reported data, and were quite surprised that such evaluation and manipulation of data required special training and knowledge. Some libraries were not organized for ongoing data collection and analysis of vendor statistics: it was unclear who was responsible for such efforts and whether resources were available to support these efforts. And finally, most libraries simply had no management information system (even in the most basic sense of the word) for organizing, analyzing, and reporting such data. The study team found that, in general, libraries were not prepared to commit the necessary resources, staff time, training, and effort into the evaluation..$^{20}$

Thus, one difficulty in some of the discussions with the vendors was a lack of knowledge and skills on the part of the librarians in using and analyzing the data. The fact of the matter is that both the library community and the vendor community have much to learn in terms of understanding how best to define, collect, analyze, report, and validate such statistics. For their part, many libraries simply do not have a culture of evaluation that supports the assessment effort needed to use vendor-based statistics successfully. ${ }^{21}$ Organizational development, staff training, and strategic planning for exploiting such data on the part of libraries will be key components in moving forward in this area.

Several organizations in library and vendor communities, national and international bodies, are currently working in this area. Although these initiatives do not overlap exactly in terms of goals and scopes, there is a danger that they may result in conflicting reporting requirements. Specific ways to coordinate and encourage cooperation have yet to be developed. Indeed, the number and range of organizations interested in developing standardized statistics is significant.

From the vendors' point of view, it is impossible to respond to multiple and conflicting requests for data from the library community. As one vendor commented, until the library community can decide how best it wants the data defined, collected, validated, and reported, vendors cannot provide endless responses to users in terms of offering "customized" data sets. Thus, to some degree, the members of the library community must continue to work among themselves to reach such an agreement regarding these standards.

In addition, different types of libraries (academic, school, public, special, etc.) need to reconsider the degree to which they think their needs are unique to their particular settings. A "full-text download" is not going to vary across type of library. Those librarians who argue that they have unique or special data needs simply support the view of some vendors that it is impossible to provide multiple data types, defined differently, for different libraries. And little progress will be made on standardizing these statistics. The members of the library community must work together in the development of such standards, definitions, and reporting.

Both vendors and the library community need to realize that the development, testing, refinement, and standardization of vendor-based statistics is an ongoing process. Given the changes in technology, database structures, and other factors, the life span of the statistics may be short (compared with more traditional library statistics). Thus, being able to have longitudinal data from vendors may be difficult and there will be a need to be much more pragmatic as to the availability and use of vendor-based statistics. Establishment of the 
National Clearinghouse for Library and Information Center Networked Statistics (http://www.ii.fsu.edu) at Florida State University's Information Use Management and Policy Institute will play a coordinating role in the collection, use, and analysis of network data sources including, but not limited to, database vendor statistics. The clearinghouse will facilitate the cross-fertilization of the various efforts thus far to build on each other and integrate activities for meaningful library assessment in support of decision making and analysis.
One important accomplishment during the project was the initiation of conversations and cooperation with major database vendors. Currently, library leadership in this area is diffuse and lacks coordination. Work needs to continue, especially in the standardization of key usage statistics, data delivery, and better documentation of definitions and reporting procedures. An ongoing, more formalized mechanism is essential to ensure that such meetings take place, progress is made, and better standards for vendorbased statistics are developed.

\section{Notes}

1. Association of Research Libraries, ARL Supplementary Statistics 2000-2001 (ARL, Washington, D. C., 2002). Available online from: <http://www.arl.org/stats/sup/sup01.pdf>.

2. It is quite possible that the users may not even realize that the library evaluated the electronic sources and negotiated the licensing contracts. Most electronic databases validate legitimate user log-ins by examining the origination IP (Internet protocol) addresses included in the requests. Users can bypass the library Web site when they access external electronic databases as long as they use the computers carrying the legitimate IP addresses. For remote users using Internet service providers (ISPs), they may need to set up a proxy server that allows them to access electronic databases. The proxy information allows them to use the institution's IP addresses.

3. The arrows denote movement of information contents and location of user access. In the depiction of the traditional library, materials reside in library premises and users come to the library to use the collection. On the other hand, in the networked library, not all of the library collection resides within the library's physical boundaries. Also, part of user access occurs outside the library, as in the case of access to most subscription-based, electronic-licensed materials.

4. These include off-site storage facilities libraries use to reduce the cost of warehousing less frequently requested materials.

5. The authors are not suggesting here that only external, licensed materials are of concern to scholars and libraries. They acknowledge that other freely available or community-based services are being heavily used by academic users.

6. There can be a set of arbitrary limitations such as simultaneous log-in limits, but they do not originate from the characteristics of the electronic format.

7. International Coalition of Library Consortia, "Guidelines for Statistical Measures of Usage of Web-Based Information Resources" (ICOLC, 2001). [revised December 2001]. Available online from http://www.library.yale.edu/consortia/2001webstats.htm.

8. There is a large and diverse number of electronic content providers in the market, and it is difficult to describe them collectively. The term "database vendors" is used in this article to denote various content providers such as traditional journal publishers providing electronic counterparts, aggregators of full-text journals and reference databases (e.g., JSTOR, Project Muse, Ebsco, Gale, ProQuest), electronic book providers (e.g., Questia, netLibrary), and so on.

9. John Carlo Bertot, Charles R. McClure, and Joe Ryan, Statistics and Performance Measures for Public Library Networked Services (Chicago: ALA, 2001).

10. Carol Tenopir and Eleanor Read, "Patterns of Use and Usage Factors for Online Databases in Academic Libraries," College \& Research Libraries 61 (May 2000): 234-46.

11. Deborah D. Blecic, Joan B. Fiscella, and Stephen E. Wiberley Jr., "The Measurement of Use of Web-based Information Resources: An Early Look at Vendor-supplied Data," College $\mathcal{E}$ Research Libraries 62 (Sept. 2001): 434-53.

12. Sally A. Rogers, "Electronic Journal Usage at Ohio State University," College E Research Libraries 62 (Jan. 2001): 25-34.

13. Kathleen Bauer, "Indexes as Tools for Measuring Usage of Print and Electronic Resources," College \& Research Libraries 62 (Jan. 2001): 36-42.

14. Charles R. McClure and John Carlo Bertot, ed., Evaluating Networked Information Services: Techniques, Policy, and Issues (Medford, N.J.: American Society for Information Science and Tech- 
nology, 2001).

15. The meeting served as the planning session for the project that later became known as the ARL E-Metrics Project. Thirty-five ARL institutions were represented at the meeting. A survey questionnaire was sent out before the meeting, and twenty-one libraries responded. The survey contained four open-ended questions on the data needs for electronic information resources, the status of data collections at participating libraries, and the expectation for the meeting. The summary presentation during the meeting (in the Microsoft Powerpoint format) is available online from http://www.arl.org/stats/newmeas/scottsdale/jeffshim/.

16. Bertot, McClure, and Ryan, Statistics and Performance Measures for Public Library Networked Services.

17. Additional meetings between vendors and other members of the library community regarding statistics have occurred at ALA midwinter 2001 and 2002. These meetings also have attempted to better coordinate and validate vendor-based statistics that are being reported to libraries. The National Commission on Libraries and Information Science has sponsored these meetings and summaries, as well as other related reports, and is available online from http:// www.nclis.gov/statsurv/statsurv.html.

18. An interim Phase I report describing current practices of participating ARL member libraries related to network statistics and measures was issued November 7, 2000, and is available online from http://www.arl.org/stats/newmeas/emetrics/index.html.

19. Wonsik Shim, Charles R. McClure, Bruce T. Fraser, and John Carlo Bertot, Data Collection Manual for Academic and Research Library Network Statistics and Performance Measures (Tallahassee, Fla.: Information Use Management and Policy Institute (Dec. 2001); also available from the ARL online from http://www.arl.org/stats/newmeas/emetrics/index.html.

20. As part of the E-Metrics Project, the study team produced three PowerPoint presentations about preparing the organization for evaluation, the importance of evaluation and use of vendor statistics, and an overview/introduction to the recommended statistics. These presentations are available from the ARL, in Washington, D.C. and from http://www.arl.org/stats/newmeas/ emetrics/index.html.

21. Amos Lakos, "The Missing Ingredient: Culture of Assessment in Libraries," Performance Measurement and Metrics: The International Journal for Library and Information Services 1 (1): 3-7. 